\title{
Signals of strong electronic correlation in ion scattering processes
}

\author{
F. Bonetto, ${ }^{1,2, *}$ C. Gonzalez, ${ }^{3}$ and E. C. Goldberg ${ }^{1,2}$ \\ ${ }^{1}$ Instituto de Física del Litoral (CONICET-UNL), Güemes 3450, S3000GLN Santa Fe, Argentina \\ ${ }^{2}$ Departamento de Ingeniería de Materiales, Facultad de Ingeniería Química, Universidad Nacional del Litoral, Santiago del Estero 2829, \\ S3000AOM, Santa Fe, Argentina \\ ${ }^{3}$ Departmento de Electronica y Tecnologia de Computadores, Facultad de Ciencias, Campus de Fuente Nueva \& CITIC, Campus de \\ Aynadamar, Universitdad de Granada, E-18071 Granada, Spain
}

(Received 29 February 2016; revised manuscript received 22 April 2016; published 26 May 2016)

\begin{abstract}
Previous measurements of neutral atom fractions for $\mathrm{Sr}^{+}$scattered by gold polycrystalline surfaces show a singular dependence with the target temperature. There is still not a theoretical model that can properly describe the magnitude and the temperature dependence of the neutralization probabilities found. Here, we applied a first-principles quantum-mechanical theoretical formalism to describe the time-dependent scattering process. Three different electronic correlation approaches consistent with the system analyzed are used: (i) the spinless approach, where two charge channels are considered $\left(\mathrm{Sr}^{0}\right.$ and $\left.\mathrm{Sr}^{+}\right)$and the spin degeneration is neglected; (ii) the infinite-U approach, with the same charge channels $\left(\mathrm{Sr}^{0}\right.$ and $\left.\mathrm{Sr}^{+}\right)$but considering the spin degeneration; and (iii) the finite-U approach, where the first ionization and second ionization energy levels are considered very, but finitely, separated. Neutral fraction magnitudes and temperature dependence are better described by the finite- $U$ approach, indicating that $e$-correlation plays a significant role in charge-transfer processes. However, none of them is able to explain the nonmonotonous temperature dependence experimentally obtained. Here, we suggest that small changes in the surface work function introduced by the target heating, and possibly not detected by experimental standard methods, could be responsible for that singular behavior. Additionally, we apply the same theoretical model using the infinite- $U$ approximation for the $\mathrm{Mg}$-Au system, obtaining an excellent description of the experimental neutral fractions measured.
\end{abstract}

DOI: 10.1103/PhysRevB.93.195439

\section{INTRODUCTION}

It is accepted that Kondo physics [1] may be observed in ion scattering experiments by choosing appropriate projectile ions and very low velocities [2,3]. These experiments can actually be accomplished. The ion fractions of $\mathrm{Sr}^{+}, \mathrm{Mg}^{+}$, and $\mathrm{Ga}^{+}$backscattered by a polycrystalline Au surface have been measured by the group of Yarmoff [4,5]. They found different temperature dependencies of the neutral fraction that might be suggesting the presence of strong electron correlation effects. $\mathrm{Up}$ to now there is not a conclusive theoretical description of these experimental results that allows us to understand how the electron-electron interaction in the atomic localized state is affecting the charge transfer and its dependence with the surface temperature. Two previous articles, by the same authors, present a theoretical description of He and Yarmoff's experiments with a limited success [6,7]. They used a model calculation based on pseudoparticle operators [8] within the finite- $U$ noncrossing approximation $[2,9,10]$. They obtained a neutral fraction quite strongly dependent on temperature within an approximation they refer as the " $U=0$ limit" ( $U$ represents the electron repulsion energy in the atomic state) but that actually corresponds to an infinite $U$ limit approximation [6]. Under the finite- $U$ approximation they a found a slight negative temperature dependence of the neutral fraction that strongly depends on the surface work function. The neutral fraction found in Refs. [6,7] failed in reproducing both the neutral fraction temperature dependence and neutral fractions measured. On the other hand, the neutral fractions of $1-\mathrm{keV}$

*Corresponding author: bonetto@santafe-conicet.gov.ar incoming $\mathrm{Mg}$ ions calculated in [7] satisfactorily reproduce the experimental results.

We believe that Yarmoff's experiments deserve more effort in the theoretical analysis since they represent an excellent opportunity to obtain important physical information from a theoretical model able to properly describe the experimental data obtained.

In the present work we study the charge transfer between positive ions of $\mathrm{Sr}$ and $\mathrm{Mg}$ and an Au surface at low incoming energies. Both systems are described by an Anderson Hamiltonian projected over the most probable atomic configurations and the Hamiltonian terms are calculated by using our bondpair model [11], which has proved to be successful in a great variety of interacting systems (see for example [12-17] and references therein).

Taking into account the first and second ionization potentials of the isolated $\mathrm{Sr}$ atom (5.7 and $11 \mathrm{eV}$, respectively), jointly with the energy level shifts (due to image potential) and the work function of the gold surface $(5.1 \mathrm{eV})$; three charge state configurations are expected to be active in the chargetransfer process: neutral $\left(\mathrm{Sr}^{0}\right)$, single $\left(\mathrm{Sr}^{+}\right)$, and double $\left(\mathrm{Sr}^{++}\right)$ ionized atoms. The different atomic charge state probabilities are calculated by employing the appropriate Green-Keldysh functions [18] solved by means of the equation of motion method (EOM), using a strict second order in the coupling term with the band states [19].

Both our $a b$ initio calculation of the Anderson Hamiltonian terms and the Green functions solved by using the EOM method, used to determine the different atomic charge states, are the most important differences with the theoretical model used in Ref. [6]. Moreover, in Ref. [6] the authors mentioned them as the two main points to be improved in their work. 
The adiabatic evolution of the atom spectral densities and the valence occupation as a function of the target temperature are also studied in order to provide a clear picture of the correlation regimes taking place at different atom-surface distances.

In the case of $\mathrm{Mg}$, the first $(7.65 \mathrm{eV})$ and second $(15.04 \mathrm{eV})$ ionization potentials of the isolated $\mathrm{Mg}$ atom suggest a large neutralization and a negligible probability of having double ionized species in the interaction of $\mathrm{Mg}$ with an $\mathrm{Au}$ surface. Then, the infinite- $U$ limit should be appropriate in this case. In the present work we show that the calculated neutral fraction using the infinite- $U$ limit approximation effectively describes the measured neutral fraction temperature dependence for 1 and 2-keV incoming energies.

\section{THEORETICAL ASPECTS}

Strontium and magnesium neutral atoms have full electronic inner shells with two $s$-valence electrons in the outermost occupied shell. The possible atomic valence states correspond to neutral, single, and double ionized atomic configurations represented by $|\uparrow, \downarrow\rangle$ (neutral); $|\uparrow, 0\rangle$ and $|0, \downarrow\rangle$ (single ionized); and $|0,0\rangle$ (double ionized), respectively.

The Anderson Hamiltonian projected over this configuration space can be written as

$$
\begin{aligned}
H= & \sum_{k \sigma} \varepsilon_{k} \hat{n}_{k \sigma}+\varepsilon_{0}|0,0\rangle\langle 0,0|+\varepsilon_{1}[|\uparrow, 0\rangle\langle\uparrow, 0|+| 0, \downarrow\rangle\langle 0, \downarrow|] \\
& +\varepsilon_{2}|\uparrow, \downarrow\rangle\langle\uparrow, \downarrow| \\
& +\sum_{k}\left[V_{k s} \hat{c}_{k \uparrow}^{\dagger}|0,0\rangle\langle\uparrow, 0|+\text { H.c. }\right] \\
& +\sum_{k}\left[V_{k s} \hat{c}_{k \downarrow}^{\dagger}|0,0\rangle\langle 0, \downarrow|+\text { H.c. }\right] \\
& -\sum_{k}\left[V_{k s} \hat{c}_{k \downarrow}^{\dagger}|\uparrow, 0\rangle\langle\uparrow, \downarrow|+\text { H.c. }\right] \\
& +\sum_{k}\left[V_{k s} \hat{c}_{k \uparrow}^{\dagger}|0, \downarrow\rangle\langle\uparrow, \downarrow|+\text { H.c. }\right] .
\end{aligned}
$$
is

The normalization condition of the selected atomic space

$$
|0,0\rangle\langle 0,0|+| \uparrow, 0\rangle\langle\uparrow, 0|+| 0, \downarrow\rangle\langle 0, \downarrow|+| \uparrow, \downarrow\rangle\langle\uparrow, \downarrow|=1 .
$$

The infinite- $U$ limit approximation corresponds to disregarding the zero electron configuration $|0,0\rangle$ in Eq. (1). More specifically, the charge fluctuation from one to zero valence electrons in the atom is neglected (in the present case, this means a second ionization energy tending to $-\infty$ ). The spinless approximation corresponds also to a charge fluctuation from one to two electrons in the atomic state where the spin degeneration is completely ignored during the charge-transfer process.

The $k$ index denotes band states, $V_{k s}$ is the coupling between band states and the $s$-orbital of the atom. The total energies of the atomic configurations, $\varepsilon_{0}=E_{\mathrm{tot}}(|0,0\rangle), \varepsilon_{1}=$ $E_{\text {tot }}(|\uparrow, 0\rangle)=E_{\text {tot }}(|0, \downarrow\rangle), \varepsilon_{2}=E_{\text {tot }}(|\uparrow, \downarrow\rangle)$ and the coupling term $V_{k s}$ depend on the position $\vec{R}$ of the atom respect to the surface. At infinite distance it is verified that

$$
\begin{aligned}
& I_{1}=E_{\mathrm{tot}}(|\uparrow, 0\rangle)-E_{\mathrm{tot}}(|\uparrow, \downarrow\rangle), \\
& I_{2}=E_{\mathrm{tot}}(|0,0\rangle)-E_{\mathrm{tot}}(|\uparrow, 0\rangle),
\end{aligned}
$$

$I_{1}$ and $I_{2}$ being the first and second ionization potentials of the projectile atom respectively.

In the scattering process, these Hamiltonian terms depend on time due to the atom's motion with a finite velocity $\vec{v}$ along the in and out trajectories defined by $\vec{R}=\vec{R}_{t p}+\vec{v}|t|$, where $\vec{R}_{t p}$ is the turning point.

In the dynamical process, the time-dependent probabilities of the different atomic charge configurations, $n_{0}(t) \equiv\langle\mid 0,0\rangle\langle 0,0 \mid\rangle_{t}, \quad n_{1}(t) \equiv\langle\mid \uparrow, 0\rangle\langle\uparrow, 0 \mid\rangle_{t}=\langle\mid 0, \downarrow\rangle\langle 0, \downarrow \mid\rangle_{t}$, and $n_{2}(t)=\langle\mid \uparrow, \downarrow\rangle\langle\uparrow, \downarrow \mid\rangle_{t}$, are obtained from the Keldysh Green functions:

$$
\begin{gathered}
F_{\uparrow}\left(t, t^{\prime}\right)=i\left\langle\left[|\uparrow, 0\rangle\left\langle 0,\left.0\right|_{t^{\prime}}, \mid 0,0\right\rangle\left\langle\uparrow,\left.0\right|_{t}\right]\right\rangle,\right. \\
F_{\uparrow \downarrow}^{\uparrow}\left(t, t^{\prime}\right)=i\left\langle\left[|\uparrow, \downarrow\rangle\left\langle\uparrow,\left.0\right|_{t^{\prime}}, \mid \uparrow, 0\right\rangle\left\langle\uparrow,\left.\downarrow\right|_{t}\right]\right\rangle,\right. \\
G_{\uparrow}\left(t, t^{\prime}\right)=i \theta\left(t^{\prime}-t\right)\left\langle\left\{|\uparrow, 0\rangle\left\langle 0,\left.0\right|_{t^{\prime}}, \mid 0,0\right\rangle\left\langle\uparrow,\left.0\right|_{t}\right\}\right\rangle,\right. \\
G_{\uparrow \downarrow}^{\uparrow}\left(t, t^{\prime}\right)=i \theta\left(t^{\prime}-t\right)\left\langle\left\{|\uparrow, \downarrow\rangle\left\langle\uparrow,\left.0\right|_{t^{\prime}}, \mid \uparrow, 0\right\rangle\left\langle\uparrow,\left.\downarrow\right|_{t}\right\}\right\rangle .\right.
\end{gathered}
$$

The average \langle\rangle is taken in the wave function that describes the interacting system in the Heisenberg picture, and the [] symbol in Eqs. (3) and (4) denotes a commutator. The Green functions (5) and (6) are the typical advanced Green functions written in terms of the projection operators.

The Green functions, Eqs. (3)-(6), are calculated by using the method based on the equations of motion closed within a strict second order in the coupling term. Details are given in Ref. [19]. The initial conditions for the scattering of $\mathrm{Sr}^{+}$by a gold surface correspond to a local magnetic moment on the atom:

$$
\begin{aligned}
\langle\mid \uparrow, 0\rangle\langle\uparrow, 0 \mid\rangle_{-\infty}+ & \langle\mid 0, \downarrow\rangle\langle 0, \downarrow \mid\rangle_{-\infty}=1 \quad \text { and } \\
\langle\mid \uparrow, \downarrow\rangle\langle\uparrow, \downarrow \mid\rangle_{-\infty} & =0 .
\end{aligned}
$$

In the stationary case, after Fourier transforming the Green functions (5) and (6), we obtain $G_{\uparrow}(\omega)$ and $G_{\uparrow \downarrow}^{\uparrow}(\omega)$. They allow us to determine the single and double occupation of the atom valence state in the following way:

$$
\begin{aligned}
& n_{1}=\frac{1}{\pi} \int_{-\infty}^{\infty} d \omega f_{<}(\omega) \operatorname{Im} G_{\uparrow}(\omega), \\
& n_{2}=\frac{1}{\pi} \int_{-\infty}^{\infty} d \omega f_{<}(\omega) \operatorname{Im} G_{\uparrow \downarrow}^{\uparrow}(\omega) .
\end{aligned}
$$

In Eqs. (7), $f_{<}(\omega)=\frac{1}{1+e^{\left(\omega-E_{F}\right) / k_{B} T}}$ is the Fermi distribution with a temperature $T$, and Fermi energy $E_{F}$. From the Green functions in the projection operator language we can recover the Green function $G_{\sigma}(\omega)$ written in terms of the fermionic operators $\hat{c}_{s \sigma}^{+}\left(\hat{c}_{s \sigma}\right)$ that creates (destroys) an electron in the 
atomic orbital with a spin projection $\sigma$ [1]:

$$
\begin{aligned}
G(\omega)= & \frac{1-\left\langle\hat{n}_{a \bar{\sigma}}\right\rangle}{\tilde{\omega}-\varepsilon_{I}-\Sigma_{0}(\omega)+\frac{U \Sigma_{<}(\omega)}{\tilde{\omega}-\varepsilon_{I}-U-\Sigma_{0}(\omega)-\Sigma_{1}(\omega)}}+\frac{\left\langle\hat{n}_{a \bar{\sigma}}\right\rangle}{\tilde{\omega}-\varepsilon_{I}-U-\Sigma_{0}(\omega)+\frac{U\left[\Sigma_{<}(\omega)-\Sigma_{1}(\omega)\right]}{\tilde{\omega}-\varepsilon_{I}-\Sigma_{0}(\omega)-\Sigma_{1}(\omega)}} \\
& +\frac{U \xi(\omega)}{\left[\tilde{\omega}-\varepsilon_{I}-\Sigma_{0}(\omega)\right]\left[\tilde{\omega}-\varepsilon_{I}-U-\Sigma_{0}(\omega)-\Sigma_{1}(\omega)\right]+U \Sigma_{<}(\omega)} .
\end{aligned}
$$

In Eq. (8) we have introduced the following functions:

$$
\begin{aligned}
\sum_{0}(\omega)= & \sum_{k} \frac{\left|V_{k s}\right|^{2}}{\omega-\varepsilon_{k}-i \eta}, \\
\sum_{1}(\omega)= & \sum_{k} \frac{\left|V_{k s}\right|^{2}}{\omega+\varepsilon_{k}-\varepsilon_{A}-i \eta}+\sum_{k} \frac{\left|V_{k s}\right|^{2}}{\omega-\varepsilon_{k}-i \eta}, \\
\sum_{<}(\omega)= & \sum_{k} \frac{\left|V_{k s}\right|^{2} f_{<}\left(\varepsilon_{k}\right)}{\omega+\varepsilon_{k}-\varepsilon_{A}-i \eta}+\sum_{k} \frac{\left|V_{k s}\right|^{2} f_{<}\left(\varepsilon_{k}\right)}{\omega-\varepsilon_{k}-i \eta}, \\
\xi(\omega)= & \sum_{k} V_{k s} \frac{\left\langle\hat{c}_{s-\sigma}^{+} \hat{c}_{k-\sigma}\right\rangle}{\omega-\varepsilon_{k}-i \eta} \\
& -\sum_{k} V_{k-\sigma} \frac{\left\langle\hat{c}_{k-\sigma}^{+} \hat{c}_{s-\sigma}\right\rangle}{\omega+\varepsilon_{k}-\varepsilon_{A}-i \eta} .
\end{aligned}
$$

The $\varepsilon_{I}$ energy is defined as $\varepsilon_{I}=\varepsilon_{1}-\varepsilon_{0}$ and $\varepsilon_{A}=2 \varepsilon_{I}+U$. The total $s$-orbital occupation per $\operatorname{spin}\left\langle\hat{n}_{s \sigma}\right\rangle$ can be obtained straightforwardly from the Green function (8) as

$$
\left\langle n_{s \sigma}\right\rangle=\frac{1}{\pi} \int_{-\infty}^{\infty} d \omega f_{<}(\omega) \operatorname{Im} G(\omega)=n_{1}+n_{2} .
$$

The importance of working with the Green functions (5) and (6) instead of Green function (8) resides in that the latter does not permit us to easily discriminate the single and double occupation probabilities [Eqs. (7)].

\section{A. Atom-surface interaction: Bond-pair model}

The bond-pair model has been successfully used in many different projectile-target combinations [12-17]. The atom energy and the hopping terms are obtained from a model Hamiltonian for the atom-surface adiabatic interaction based on both the localized atom-atom interactions and the extended features of the surface states [11]. A linear combination of atomic orbitals (LCAO) is used to expand the surface band states, and a mean-field approximation of the two-electron interaction terms are performed. The effect of the long-range interactions is introduced in the case of metal surfaces by considering the image potential defining the energy level shifts for large normal distances $(z)$ to the surface. The energy level of the first electron in the $5 s$ orbital of the $\mathrm{Sr}$ atom is

$$
\varepsilon_{I}(R)=\tilde{\varepsilon}_{I}(R)+V_{i m}^{S r^{++}}(z)
$$

and for the second electron, $\varepsilon_{a f}=\varepsilon_{I}+U$, is

$$
\varepsilon_{a f}(R)=\tilde{\varepsilon}_{a f}(R)+V_{i m}^{S r^{+}}(z) .
$$

In Eqs. (10) and (11), $\tilde{\varepsilon}_{I}(R)$ and $\tilde{\varepsilon}_{a f}(R)$ take into account the short-range contributions to the energy-level variation with the atom-surface distance caused by the overlap and meanfield electrostatic interactions [11]. Far from the surface, the energy shift of the double ionized atomic configuration is four times the corresponding energy shift for the single ionized configuration.

The image potential is matched at a distance $z_{0}$ as follows:

$$
\begin{aligned}
\varepsilon_{I}(R) & =\tilde{\varepsilon}_{I}(R)+V_{i m}^{S r^{++}}(z), \text { where } V_{i m}^{S r^{++}}(z) \\
& =\left\{\begin{array}{l}
\frac{3}{4\left|z-z_{i m}\right|} z>z_{0} \\
\frac{3}{4\left|z_{0}-z_{i m}\right|} z<z_{0}
\end{array}\right. \\
\varepsilon_{a f}(R) & =\tilde{\varepsilon}_{a f}(R)+V_{i m}^{S r^{+}}(z), \text { where } V_{i m}^{S r^{+}}(z) \\
& =\left\{\begin{array}{l}
\frac{1}{4\left|z-z_{i m}\right|} z>z_{0} \\
\frac{1}{4\left|z_{0}-z_{i m}\right|} z<z_{0}
\end{array}\right.
\end{aligned}
$$

The image plane position for $\mathrm{Au}(100)$ surface is $z_{i m}=$ 1.6 a.u. [20], and $z_{0}$ is chosen equal to 8 a.u. [11].

The model calculation is based on an expansion of the solid states $\phi_{\vec{k}}(\vec{r})$ in the atomic states $\varphi_{\alpha}\left(\vec{r}-\vec{R}_{i}\right)$ centered on the different atoms of the solid ( $\alpha$ denotes the orbital type and $\vec{R}_{i}$ the position relative to the scatter atom). In this way, the coupling term $V_{\vec{k} s}$ can be written as

$$
V_{\vec{k} s}(\vec{R})=\sum_{\alpha, i} c_{\alpha, i}^{\vec{k}} V_{\alpha i, s}\left(\vec{R}-\vec{R}_{i}\right),
$$

where $V_{\alpha i, s}\left(\vec{R}-\vec{R}_{i}\right)$ is the atomic coupling between the $\alpha$ states of the $i$ atom of the solid and the $s$ state of the projectile positioned at the distance $\vec{R}$ from the scatter atom, calculated by using a mean-field approximation and a symmetrically orthonormalized atomic basis set in the space of the dimmer projectile-solid atom [11]. The coefficients $c_{\alpha, i}^{\vec{k}}$ in the expansion (14) define the density matrix of the solid given by the expression

$$
\rho_{i, j, \alpha, \beta}(\varepsilon)=\sum_{\vec{k}} c_{\alpha, i}^{\vec{k}} c_{\beta, j}^{\vec{k}} \delta\left(\varepsilon-\varepsilon_{\vec{k}}\right)
$$

Thus quantities like the noninteracting self-energy $\Sigma_{0}(\omega)$ in Eqs. (9) are calculated in terms of the surface density of states [Eq. (15)] and the atom-atom coupling terms $V_{\alpha i, s}\left(\vec{R}-\vec{R}_{i}\right)$ :

$$
\begin{aligned}
\sum_{0}(\omega)= & \sum_{i, \alpha, j, \beta} V_{\alpha, s}\left(\vec{R}-\vec{R}_{i}\right) V_{\beta, s}\left(\vec{R}-\vec{R}_{j}\right) \\
& \times \int_{-\infty}^{\infty} d \varepsilon \frac{\rho_{i, j, \alpha, \beta}(\varepsilon)}{\omega-\varepsilon-i \eta} .
\end{aligned}
$$




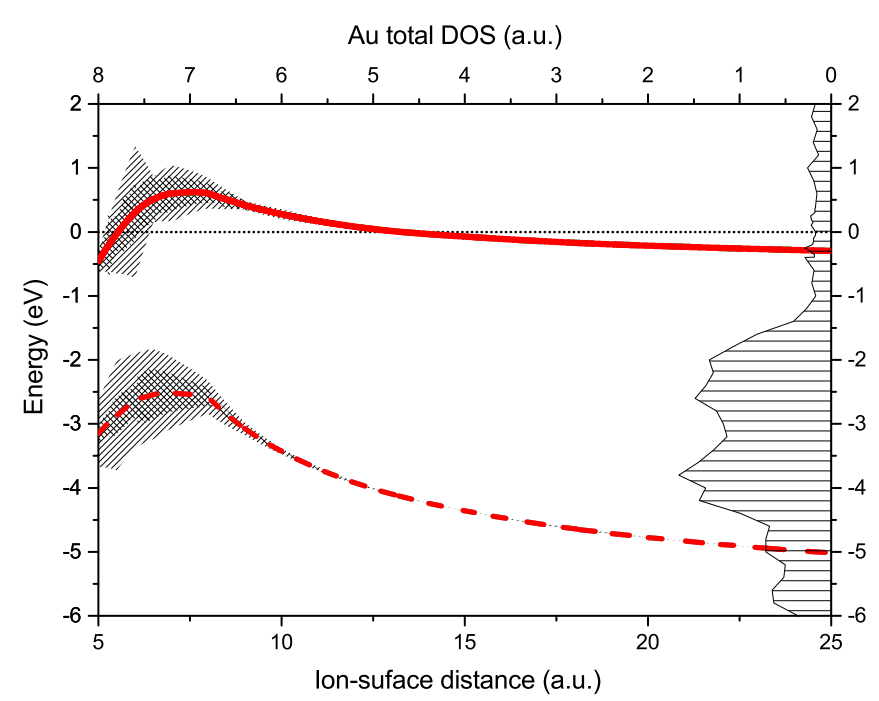

FIG. 1. Affinity (solid line) and ionization energy levels of the projectile as a function of the ion-surface distance referred to the target-surface Fermi level (dotted line). The energy-level widths $\Gamma_{0}$ (double shaded region) and $\Gamma$ (single shade region) are included. The total $\mathrm{Au}(100) \mathrm{DOS}$ (horizontal strips) is also shown.

The imaginary part of Eq. (16) is the Anderson hybridization width:

$$
\Gamma_{0}(\omega)=\pi \sum_{i, \alpha, j, \beta} V_{\alpha, s}\left(\vec{R}-\vec{R}_{i}\right) V_{\beta, s}\left(\vec{R}-\vec{R}_{j}\right) \rho_{i, j, \alpha, \beta}(\omega) .
$$

In Fig. 1, we summarize the results issued by the bond-pair model. This figure shows the one electron energy levels given by Eqs. (12) and (13), and the corresponding noninteracting level half-widths $\Gamma_{0}\left(\varepsilon_{I}\right)$ and $\Gamma_{0}\left(\varepsilon_{a f}\right)$ are represented as error bars. The level widths including the electron-electron interaction [19] are also shown in this figure,

$$
\begin{gathered}
\Gamma\left(\varepsilon_{I}\right)=\Gamma_{0}\left(\varepsilon_{I}\right)+\Gamma_{<}\left(\varepsilon_{I}\right), \\
\Gamma\left(\varepsilon_{a f}\right)=\Gamma_{0}\left(\varepsilon_{a f}\right)+\Gamma_{1}\left(\varepsilon_{a f}\right)-\Gamma_{<}\left(\varepsilon_{a f}\right),
\end{gathered}
$$

where $\Gamma_{<}$and $\Gamma_{1}$ are the imaginary parts of $\Sigma_{<}$and $\Sigma_{1}$ [Eqs. (9)] respectively.

The $\mathrm{Sr}$ and Au Gaussian basis sets provided by Huzinaga [21,22] are used to calculate the atom-atom coupling terms $V_{\alpha, s}\left(\vec{R}-\vec{R}_{i}\right)$. The density matrix $\rho_{i, j, \alpha, \beta}(\varepsilon)$ is calculated by using the localdensity approximation (LDA) [23,24].

In the present calculation we have considered only the scatter $\mathrm{Au}$ atom in the LCAO expansion of Eq. (14). This assumption is justified on the grounds that the experimental scattering geometry corresponds to a large scattering angle $\left(135^{\circ}\right)$ with an exit angle of $90^{\circ}$ relative to the target surface plane.

The distance of closest approach $\left(R_{t p}\right)$ is fixed to $z=5$ a.u., according to the large ionic radius of both atoms. Nevertheless, we found that the final neutral fraction practically does not depend on the turning point, when it is varied from 4 to 6 a.u.

The ion trajectory was assumed to be normal to the target surface during the whole collision process. However we used the correct perpendicular components of the ion velocities $\left(v_{\text {in/out }}\right)$ determined by the energy loss factor $(\alpha=0.193)$ of the
Sr-Au elastic scattering. Thus, $v_{\text {in }}=0.134 \times \sin \left(45^{\circ}\right)$ a.u.and $v_{\text {out }}=0.134 \times 0.193$ a.u.

\section{RESULTS AND DISCUSSION}

\section{A. Sr-Au system}

First, we analyze the adiabatic evolution of the interaction through the atom spectral densities and the single and double occupations [Eqs. (7)] for several atom-surface distances. In the case of the infinite- $U$ limit $\left(U \gg \Gamma_{0}\left(\varepsilon_{I}\right)+\Gamma_{0}\left(\varepsilon_{a f}\right)\right)$, different correlation regimes will take place at different projectilesurface distances depending on the ratio $r_{c}=\varepsilon_{a f} / \Gamma_{0}\left(\varepsilon_{a f}\right)[1]$.

The charge fluctuation from one to two electrons in the neutralization of $\mathrm{Sr}^{+}$corresponds in the hole picture to a fluctuation from one to zero holes. This picture is the appropriate one to analyze the double occupation in the infinite- $U$ limit. The Kondo regime occurs for $r_{c}>1$, that means $\varepsilon_{a f} \gg \Gamma_{0}$. This is an energy level well above the Fermi level and then, an occupation practically equal to 1 (hole). The mixed valence regime for $\left|r_{c}\right| \approx 0$ means an energy level $\varepsilon_{a f}$ close to the Fermi level. Finally, the empty orbital regime for $r_{c}<-1$ corresponds to $\varepsilon_{a f}$ well below the Fermi energy and thus, an occupation of zero holes. On the other hand, Fig. 1 shows that the infinite- $U$ limit becomes more valid for large distances $(z>7$ a.u. $)$

According to Eq. (7), $\rho_{2}(\omega)=(1 / \pi) \operatorname{Im} G_{\uparrow \downarrow}^{\uparrow}(\omega)$ determines the double occupation $n_{2}$. Figures 2(a)-2(c) show the spectral density $\rho_{2}(\omega)$ for several atom-surface distances $(6,7$, and 9 a.u., respectively), and different temperatures. Here, we can clearly observe that our model leads to a Kondo regime at large distances (9 a.u.), evolving to a mixed valence regime for $z=6$ a.u. We can also observe a robust resonance structure at the Fermi energy that persists up to large temperatures (near to $900 \mathrm{~K}$ ).

The double and single occupations [Eqs. (7)] as a function of temperature are shown in Fig. 3 for different distance values.

We can notice that the double electron occupation increases with temperature in the Kondo regime (the hole occupation decreases), while the opposite behavior is obtained in the mixed-valence regime $(z<8$ a.u.). This is consistent with the temperature dependence of the probability of a single electron in the $s$-valence orbital $n_{1}$, shown in Fig. 3(b).

Due to the very low velocity ( $v=0.0134$ a.u.), the neutral fraction of $\mathrm{Sr}$ atoms scattered by the Au surface is defined far from the surface along the outgoing trajectory. This means that distances larger than 8 a.u. are expected to be involved in the determination of the final charge states of Sr projectile. Therefore, according to the adiabatic results (Fig. 3), it is expected that in the scattering process the neutral fraction, $n_{2}(t \rightarrow \infty)$, increases with temperature. This is actually the result obtained, as it is shown Fig. 4. In this figure we also include the calculations based on both the spinless model and the infinite- $U$ limit approximation.

As the spinless model ( $U=0$, no spin) neglects the possibility of having electrons with spin up or down, when we go from the spinless model to the $U$ infinite model we are showing how important the spin fluctuation statistics is in this system. When we go from $U$ infinite to $U$ finite we learn how relevant the electronic correlation is by including the 


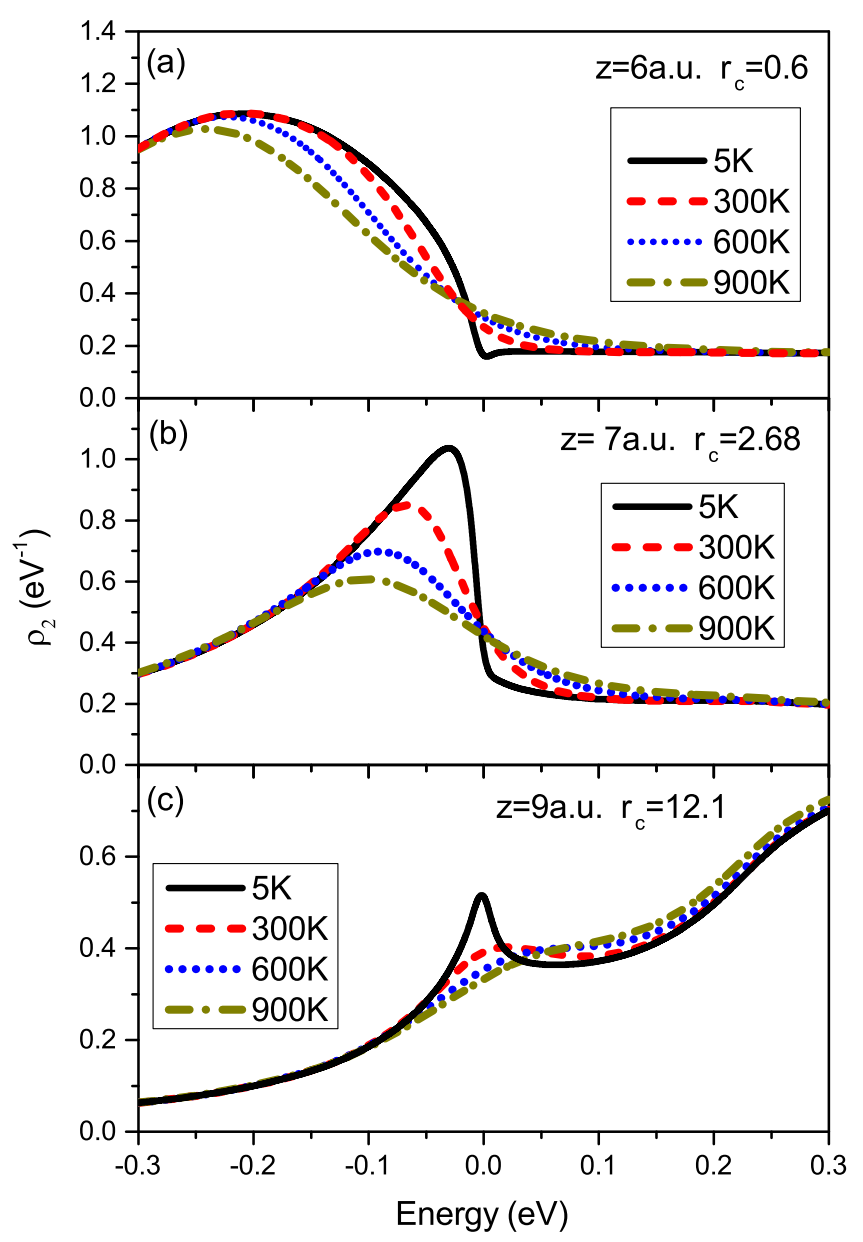

FIG. 2. Spectral density $\rho_{2}$ plotted as a function of the energy for three different ion-surface distances: 6 a.u. (a), 7 a.u. (b), and 9 a.u. (c), and four different temperatures: $5 \mathrm{~K}$ (solid line), $300 \mathrm{~K}$ (dashed line), $600 \mathrm{~K}$ (dotted line), and $900 \mathrm{~K}$ (dash-dotted line).

possibility of other electronic configurations for the projectile atom.

The differences in neutral fractions obtained under the infinite- $U$ and the spinless approaches at low temperatures can be explained in terms of the level widths in one and another approximation. In the infinite- $U$ limit, the level width is given by

$$
\Gamma^{U \rightarrow \infty}\left(\varepsilon_{a f}\right)=\operatorname{Im} \sum_{k} \frac{\left[\left|V_{k s}\right|^{2} / 2\right]\left[2-f_{<}\left(\varepsilon_{k}\right)\right]}{\varepsilon_{a f}-\varepsilon_{k}-i \eta} .
$$

When compared to $\Gamma_{0}$ [the corresponding level to the spinless approximation, Eq. (17)], we can see that the electronloss processes have the same weight in both approximations while the possibility of electron capture is lowered in the infinite- $U$ limit.

Since the atom spectral density does not depend on temperature in the spinless model, the only variation of the neutral fraction with temperature comes from the Fermi distribution, which is nearly constant in the analyzed temperature range. The fundamental difference between finite and infinite- $U$ calculations resides in the fact that the probability of double ionized $\mathrm{Sr}$ atoms along the ion trajectory is not disregarded

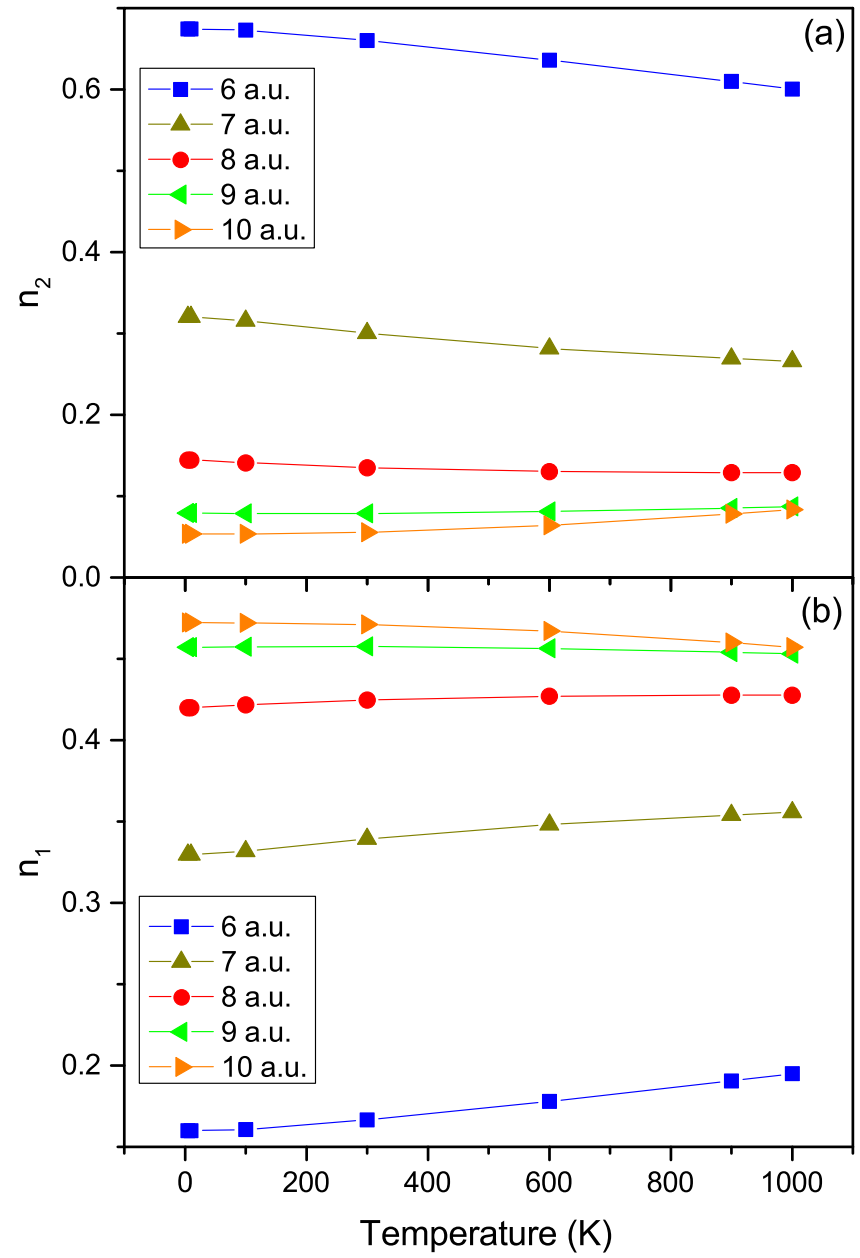

FIG. 3. The double (a) and single (b) electronic occupations are shown as a function of the target temperature for five different projectile-surface distances: 6 a.u. (squares), 7 a.u. (triangles pointing up), 8 a.u. (circles), 9 a.u. (triangles pointing left), and 10 a.u. (triangles pointing right).

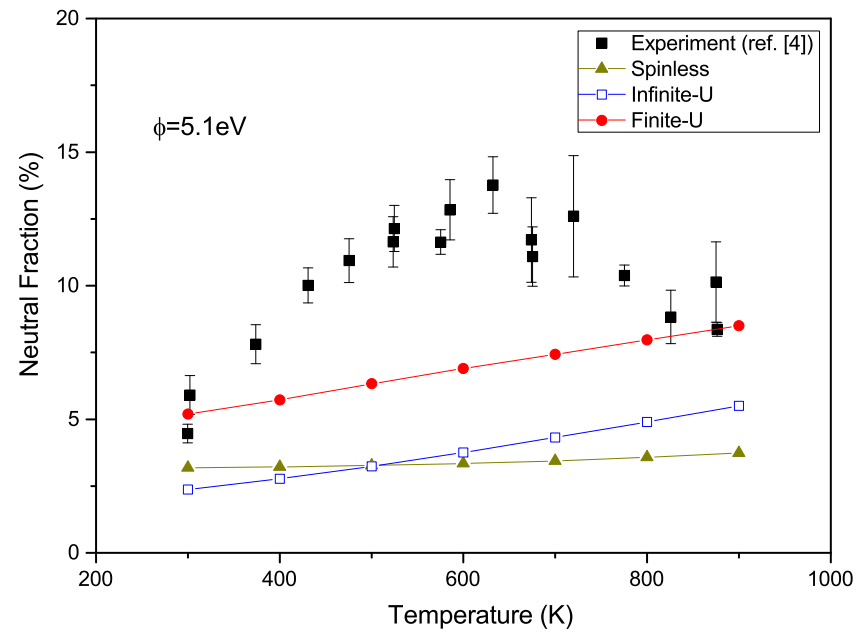

FIG. 4. Experimental (full squares) neutral fraction as a function of the target temperature contrasted with theoretical calculations under three different approximations: Spinless (triangles), infinite- $U$ limit (empty squares), and finite- $U$ approximation (circles). Measured neutral fraction magnitudes are better described by the latter (and more complete) model. 


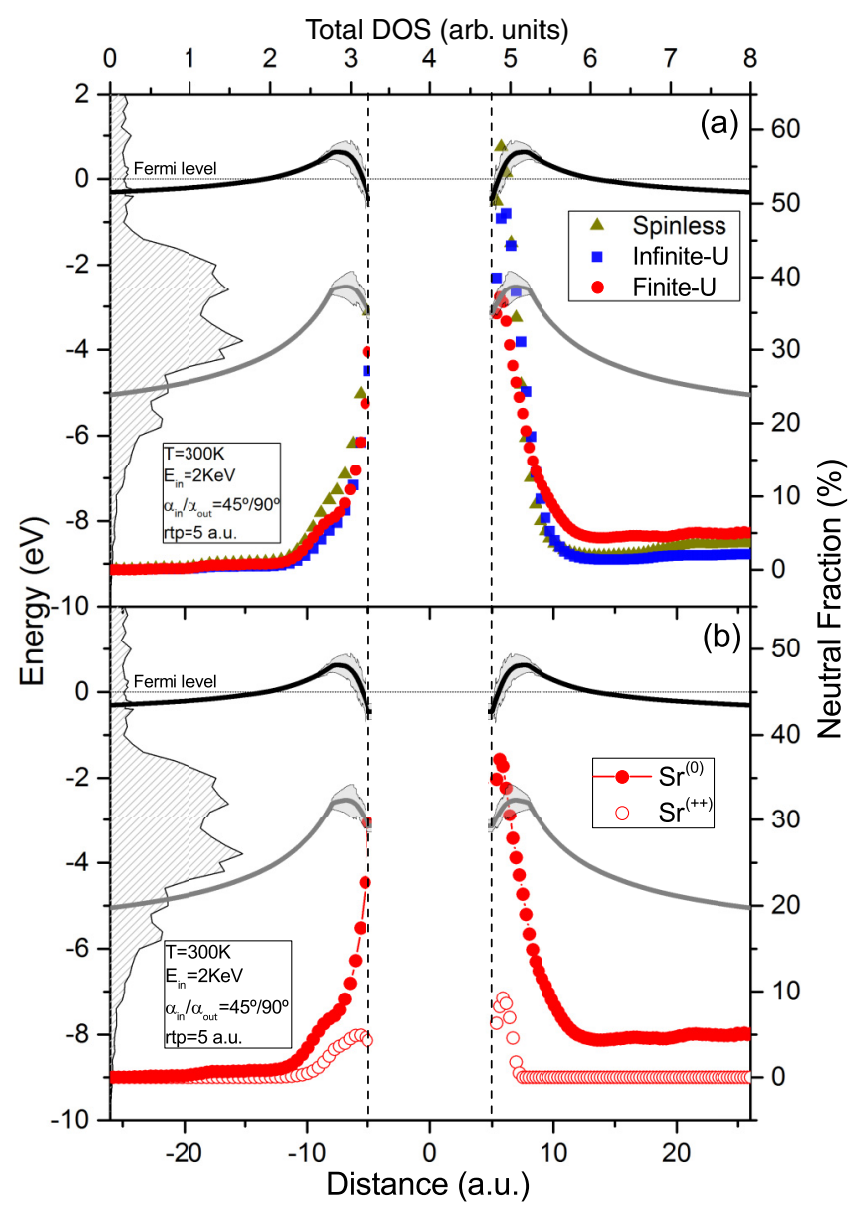

FIG. 5. (a) Neutral fraction as a function of the projectile trajectory calculated under the three approximations: spinless (triangles), infinite- $U$ limit (squares), and finite- $U$ (circles). (b) Evolution of the $\mathrm{Sr}^{0}$ and the $\mathrm{Sr}^{++}$probabilities during the collision. Negative/positive distances indicate incoming/outgoing part of the trajectory. The first and second ionization energy levels are shown with their corresponding $\Gamma_{0}$ widths. The total surface DOS is also shown (shaded region).

in the first case. In Fig. 5(a) we can see the evolution of the neutral fraction along the trajectory calculated by using the three approximations. In Fig. 5(b) the probability of occurrence of neutral $\left(\mathrm{Sr}^{0}\right)$ and double ionized $\mathrm{Sr}^{++}$are compared (both calculated under the finite- $U$ approach). Since the infinite-U limit provides a proper description of the problem for a large portion of the projectile's trajectory, it is not surprising that the temperature dependence of the neutral fraction is similar in both finite- and infinite- $U$ calculations. Our results show that the possibility of the double ionized $\mathrm{Sr}$ atoms, only appreciable at small distances from the surface, increases the neutral fraction. This is a pure correlation effect, already identified and discussed in a previous work [16].

The neutral fraction results to be quite dependent on the surface work function in this atom-surface interacting system. This is not surprising because of the proximity of the first ionization level to the Fermi level. In the next figure (Fig. 6) we can see the variation of the neutral fraction as a function of temperature for small variations of the work function (less

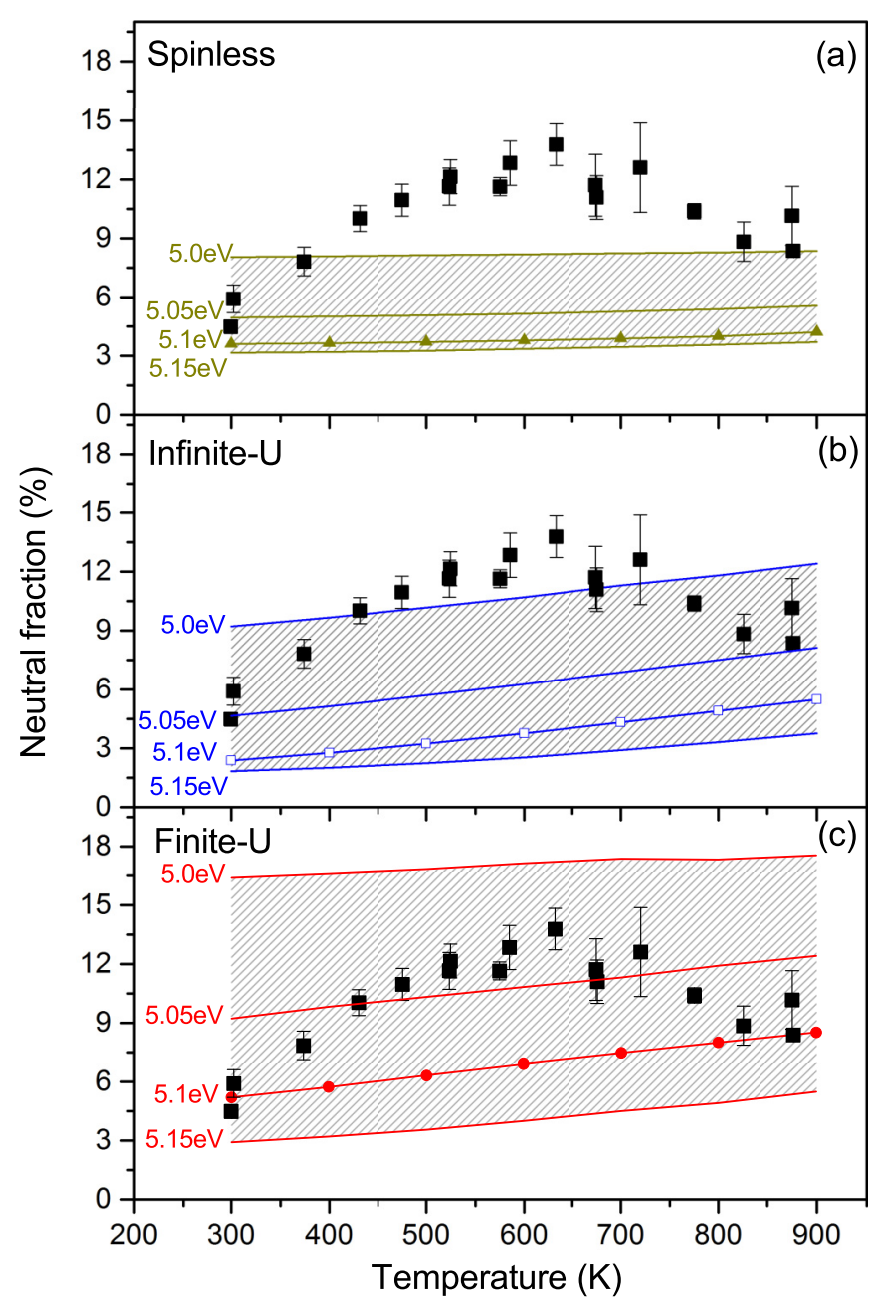

FIG. 6. (a) Calculated neutral fraction as a function of the target temperature under the three approximations above described: spinless (a), infinite- $U$ limit (b), and finite- $U$ (c). Each solid line corresponds to the calculation performed for different values of the work function around $5.1 \mathrm{eV}$ (indicated in the figure). The figure shows how sensitive the neutral fraction is to small variations in the surface work function. Moreover, typical precision in work-function measurements $(0.1 \mathrm{eV})$ introduce sufficiently large errors in the calculation to match the experimental neutral fractions obtained (shaded region, finite- $U$ approach).

than $0.1 \mathrm{eV}$ ), under the three electron-electron interaction approximations previously discussed in this work.

The finite- $U$ model calculation is the most sensitive to small variations of the surface work function. The results of Fig. 6(c) suggest that a slight nonmonotonous temperature dependence of the work function $(\Phi)$, possibly not detected within the experimental error of the work-function measurement $[25,26]$, may explain the presence of a maximum in the temperature dependence of the neutral fraction. It has been discussed a long time ago that the thermal expansion of the crystals and the thermal vibration of the atoms are mainly responsible for the temperature dependence of the work function, and that each of these two effects gives a temperature coefficient of opposite sign [27-29]. The work-function dependence on temperature extracted by fitting the results of Fig. 6(c) and shown in the 


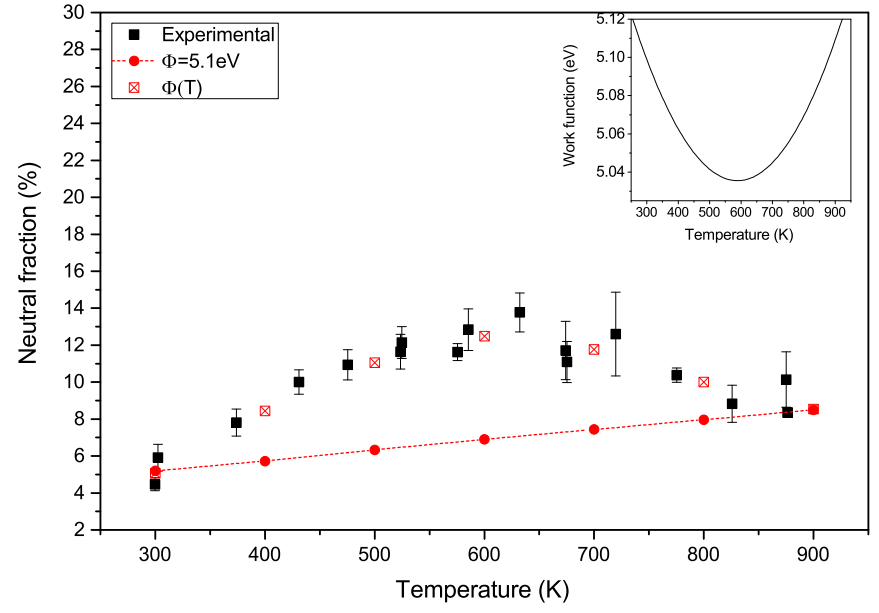

FIG. 7. Calculated neutral fraction under the finite- $U$ approach when the work function is kept constant at $\Phi=5.1 \mathrm{eV}$ (full circles) and when a small temperature dependence in the work function (inset) is assumed (empty squares with crosses). Work-function variation rates in the order of $10^{-4} \mathrm{eV} / \mathrm{K}$ are typical in metals.

inset of Fig. 7 seems to respond to these two effects, and it allows for fairly reproducing the experimental behavior with temperature of the neutral fraction (see Fig. 7).

Here, it is important to stress that the proposed temperature work-function dependence must fulfill two essential extra constraints: (i) the work function of polycrystalline $\mathrm{Au}$ at room temperature $(300 \mathrm{~K})$ is $5.1 \mathrm{eV}$ (measured independently with different methods, even with lower experimental errors) [30-32]; and (ii) the absolute deviation in the work function from $5.1 \mathrm{eV}$ should not exceed $0.1 \mathrm{eV}$ for the whole temperature range (see experimental error in work-function measurement reported in Refs. [4,5]). Thus, with these two extra constraints, it is clear from Figs. 6 and 7 that the finite- $U$ model (with $e$-correlation included) plus the work-function temperature dependence proposed is the only model capable to adequately describe the neutral fraction magnitudes and the temperature dependence experimentally observed.

\section{B. Mg-Au system}

The neutral fraction along the ion trajectory in the case of positive ions of $\mathrm{Mg}$ scattered by a gold surface is shown in Fig. 8 for two incoming energies, 1 and $2 \mathrm{keV}$, and $T=300 \mathrm{~K}$. These results were obtained by using the infinite- $U$ limit approximation, that is, only the atomic configurations with one and two electrons in the $4 s$ orbital of $\mathrm{Mg}$ are considered. The turning point is fixed in $z=4 \mathrm{a} . \mathrm{u}$. and the corresponding energy-loss factor $(0.6547)$ is considered to determine the exit velocity. The one electron energy level $\varepsilon_{a f}$ and its noninteracting width $\Gamma_{0}\left(\varepsilon_{a f}\right)$, jointly with the density of states (DOS) of $\mathrm{Au}(100)$, are also shown in Fig. 8.

We observe that the neutral fractions for the two incoming energies are very similar and practically a full neutralization $(>90 \%)$ is obtained. This result is consistent with the first ionization level well below the Fermi level along the major part of the trajectory. The level width makes possible the electron-loss processes only for distances smaller than 6 a.u. In the next figure, the calculated and measured [5] temperature

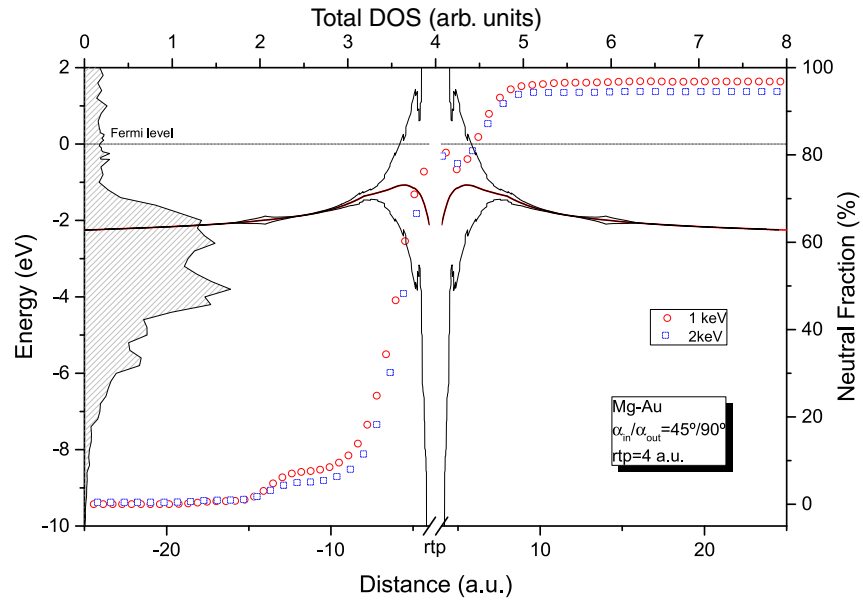

FIG. 8. Neutral fraction $\left(\mathrm{Mg}^{0}\right)$ evolution along the projectile trajectory calculatesd under the infinite- $U$ approach for two incoming energies: $1 \mathrm{keV}$ (circles) and $2 \mathrm{keV}$ (squares). An almost complete neutralization is achieved for both incoming energies consistent with an energy level far from the surface Fermi level (also shown with its corresponding $\Gamma_{0}$ width). The total $\mathrm{Au}(100)$ DOS is also included (shaded region).

dependencies of the neutral fraction are compared for both incoming energies, 1 and $2 \mathrm{keV}$.

The calculated neutral fraction is in agreement with measured values within the experimental errors. In Fig. 9 we also include the results calculated by considering the $\Phi(T)$ shown in the inset of Fig. 7. We observe that this temperature dependence of the work function only introduces a slight temperature dependence of the neutral fraction at $1 \mathrm{keV}$, which is, in some way, an obvious result since a projectile level well

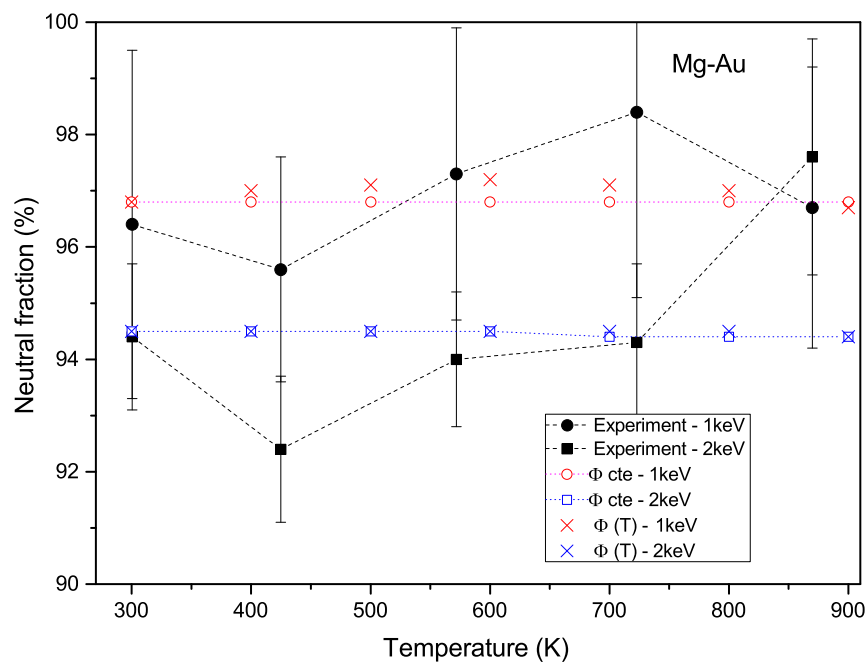

FIG. 9. Calculated (empty symbols) and experimental [5] (full symbols) are compared for two incoming energies: $1 \mathrm{keV}$ (circles) and $2 \mathrm{keV}$ (squares) when the work-function temperature is kept constant $(\Phi=5.1 \mathrm{eV})$. If the temperature dependence in the work function (inset Fig. 7) is incorporated, the calculated neutral fraction (crosses) is practically not altered. A noticeable agreement between experimental and calculated neutral fractions is achieved. 
below the Fermi energy makes the neutral fraction not sensitive to small changes of the work function.

From Fig. 8 we can also see that for $z>6$ a.u. the relation $r_{c}=\varepsilon_{a f} / \Gamma_{0}\left(\varepsilon_{a f}\right)$ is smaller than -2 , indicating an empty orbital regime and leading to practically no variation of the valence occupation with the temperature.

\section{CONCLUSIONS}

We report theoretical results about the neutralization probability of $\mathrm{Sr}^{+}$ions scattered by a gold target surface. We used three different approximations to include the electronic correlation in our model: (a) spinless, (b) the infinite- $U$ limit, and (c) the finite- $U$ limit. The proximity of $\mathrm{Sr}^{+}$and $\mathrm{Sr}^{++}$ energy levels when compared to their widths indicate that the finite- $U$ model should be more appropriate to describe the system under analysis. This speculation is indeed reinforced by the improved agreement with experimental results found in neutral fractions calculated under the finite- $U$ limit. A detailed analysis of the evolution of each probability indicates that even when the final $\mathrm{Sr}^{++}$fraction is negligible, it becomes important at low projectile-target distances and affects the final $\mathrm{Sr}^{0}$ neutral fraction.

The analysis of the adiabatic evolution of the interaction between the ion $\mathrm{Sr}^{+}$and the gold surface allows us to conclude that the Kondo regime (once considered a key factor in the anomalous neutral fraction temperature dependence obtained [4]) takes place at large distances ( $>8$ a.u.). It is found that the neutralization probability increases with temperature in the Kondo regime (the hole occupation decreases), while the opposite behavior is obtained in the mixed-valence regime.

To explain the anomalous neutral fraction temperature dependence experimentally found we proposed that slight changes, caused by the sample heating or potential sample contamination under detection limits, could be responsible for the behavior observed. We based our conjecture on two different grounds: (a) the neutral fraction in the Sr$\mathrm{Au}$ system is extremely sensitive to changes in the gold work function, even those lower than typical errors [25] in work-function measurements; (b) the necessary work-function variations $(0.1 \mathrm{meV} / \mathrm{K})$ to reproduce the observed behavior are comparable to previously reported thermal work-function shifts for metals [27]. Despite a $T^{2}$ dependence of metal work functions having been properly documented [27-29], there is no, however, any previous work that supports the temperature dependence proposed for gold surface work function in the inset of Fig. 7. Only Gd polycrystalline [27] and $\mathrm{Cu}(111)$ [28] were found to have a similar temperature dependence of that proposed for $\mathrm{Au}$.

Based on the fact that the energy level of the Mg first $s$-valence electron is found well below the gold surface Fermi level, the same theoretical formalism was applied to the $\mathrm{Mg}$-Au system under the infinite- $U$ limit. The comparison with experimental neutral fractions shows an excellent agreement, either for magnitudes and temperature dependence of the neutral fractions, for the two incoming energies considered, 1 and $2 \mathrm{keV}$. In addition, an identical work-function temperature dependence to that one proposed for the $\mathrm{Sr}$-Au system was employed for $\mathrm{Mg}$-Au. Minor changes were found when compared to theoretical calculations with a constant work function, showing that any potential slight change in the work function, either from heating or contamination sources, cannot be detected by measuring the neutral fraction in $\mathrm{Mg}-\mathrm{Au}$.

The model described above and the previous discussion could be straightforwardly extended to other alkaline-Au systems like Be-Au, Ca-Au, Ba-Au, or Ra-Au. Based on the proximity of $\mathrm{Ba}$ first ionization energy level $(5.2 \mathrm{eV})$ and gold surface Fermi level $(5.1 \mathrm{eV})$ and previous results on $\mathrm{Sr}-\mathrm{Au}$ and $\mathrm{Mg}-\mathrm{Au}$, we foresee a stronger dependence of the neutral fraction with the temperature and the work function of the $\mathrm{Au}$ surface (even stronger than the one obtained for $\mathrm{Sr}-\mathrm{Au}$ ). Unfortunately, and due to different experimental difficulties, the neutral fraction has not yet been measured in any of these systems.

\section{ACKNOWLEDGMENTS}

This work was supported by ANPCyT through PICT grants, CONICET through PIP grants, and U.N.L. through CAI+D grants.
[1] A. C. Hewson, The Kondo Problem to Heavy Fermions (Cambridge University Press, Cambridge, England, 1997).

[2] H. Shao, P. Nordlander, and D. C. Langreth, Phys. Rev. Lett. 77, 948 (1996).

[3] J. Merino and J. B. Marston, Phys. Rev. B 58, 6982 (1998).

[4] X. He and J. A. Yarmoff, Phys. Rev. Lett. 105, 176806 (2010).

[5] X. He and J. A. Yarmoff, Nucl. Instrum. Methods Phys. Res., Sect. B 269, 1195 (2011).

[6] M. Pamperin, F. X. Bronold, and H. Fehske, Phys. Rev. B 91, 035440 (2015).

[7] M. Pamperin, F. X. Bronold, and H. Fehske, Phys. Scr. 2015, 014008 (2015).

[8] P. Coleman, Phys. Rev. B 29, 3035 (1984).

[9] D. C. Langreth and P. Nordlander, Phys. Rev. B 43, 2541 (1991).

[10] H. Shao, D. C. Langreth, and P. Nordlander, Phys. Rev. B 49, 13929 (1994).
[11] P. G. Bolcatto, E. C. Goldberg, and M. C. G. Passeggi, Phys. Rev. B 58, 5007 (1998).

[12] R. A. Vidal, F. Bonetto, J. Ferrón, M. A. Romero, E. A. Garcia, and E. C. Goldberg, Surf. Sci. 605, 18 (2011).

[13] C. Meyer, F. Bonetto, R. Vidal, E. A. García, C. Gonzalez, J. Ferrón, and E. C. Goldberg, Phys. Rev. A 86, 032901 (2012).

[14] F. J. Bonetto, E. A. García, C. González, and E. C. Goldberg, J. Phys. Chem. C 118, 8359 (2014).

[15] A. Iglesias-García, F. Bonetto, R. Vidal, J. Ferrón, and E. C. Goldberg, Phys. Rev. A 89, 042702 (2014).

[16] A. Iglesias-García, E. A. García, and E. C. Goldberg, Phys. Rev. B 90, 195416 (2014).

[17] F. J. Bonetto, M. A. Romero, A. Iglesias-García, R. A. Vidal, and E. C. Goldberg, J. Phys. Chem. C 119, 3124 (2015).

[18] L. V. Keldysh, Zh. Eksp. Teor. Fiz. 47, 1515 (1964) [Sov. Phys. JETP 20, 1018 (1965)]. 
[19] M. A. Romero, F. Flores, and E. C. Goldberg, Phys. Rev. B 80, 235427 (2009).

[20] E. V. Chulkov, V. M. Silkin, and P. M. Echenique, Surf. Sci. 437, 330 (1999).

[21] S. Huzinaga, J. Andzelm, M. Klobukowsky, E. RadzioAndzelm, Y. Sakai, and H. Tatewaki, Gaussian Basis Set for Molecular Calculations (Elsevier, Amsterdam, 1984).

[22] S. Huzinaga, J. Chem. Phys. 42, 1293 (1965).

[23] P. Jelinek, H. Wang, J. P. Lewis, O. F. Sankey, and J. Ortega, Phys. Rev. B 71, 235101 (2005).

[24] J. P. Lewis, K. R. Glaesemann, G. A. Voth, J. Fritsch, A. A. Demkov, J. Ortega, and O. F. Sankey, Phys. Rev. B 64, 195103 (2001).
[25] N. D. Orf, I. D. Baikie, O. Shapira, and Y. Fink, Appl. Phys. Lett. 94, 113504 (2009).

[26] J. Holzl, F. K. Schulte, and H. Wagner, in Solid Surface Physics, edited by G. Holer (Springer-Verlag, Berlin, 1979).

[27] T. Durakiewicz, A. J. Arko, J. J. Joyce, D. P. Moore, and S. Halas, Surf. Sci. 478, 72 (2001).

[28] A. Kiejna, Surf. Sci. 178, 349 (1986).

[29] A. Kiejna, K. F. Wojciechowski, and J. Zebrowksi, J. Phys. F 9, 1361 (1979).

[30] H. B. Michaelson, J. Appl. Phys. 48, 4729 (1977).

[31] E. R. Kötz, H. Neff, and K. Müller, J. Electroanal. Chem. Interfacial Electrochem. 215, 331 (1986).

[32] K. T. Narasimha et al., Nat Nano 11, 267 (2016). 